\title{
Il libro del Cortegiano tradotto da Boscán: Nota su un lapsus maschile pro femminile
}

\author{
María de las Nieves Muñiz Muñiz ${ }^{1}$
}

Universitat de Barcelona

\begin{abstract}
Partendo dalla sostituzione di genere (le>lui) commessa da Boscán traducendo un passo del Cortegiano (III $\$ 56$ ), si studiano altre deviazioni dello stesso tenore e si riconducono all'imperfetta assimilazione dell'ideale femminile proposto da Castiglione. In appendice, Bibliografia sulla traduzione di Boscán (stampe e studi) e sulla figura della donna in Castiglione.
\end{abstract}

Parole chiave: Traduzione, Boscan, Cortegiano, Castiglione, Letteratura, Donne, Rinascimento.

\section{Abstract}

Leaving from a gender substitution (le>lui) committed by Boscan in translate a fragment of Cortegiano (III \$56), one study other meaningful deviation lines and they are led back to the imperfect assimilation of the proposed feminine ideal from Castiglione. Bibliography about the translation of Boscan (press and studies) and on the figure of the woman in Castiglione, in appendix.

Key words: Translation, Boscan, Cortegiano, Castiglione, Women Renaissance Literature.

Menéndez Pelayo ebbe a definire El Cortesano di Boscán «el mejor libro en prosa escrito en España durante el reinado de Carlos V», ${ }^{2}$ e il giudizio venne ampiamente confermato dalla tendenza a leggerlo per lungo tempo come un classico nazionale. Difatti, la maggior virtù di quella tempestiva traduzione ${ }^{3}$ consistette nel favorire il rapido assorbimento del modello proposto da Casti-

1. Queste pagine nascono dalla ricerca condotta nel quadro del Programma sulle traduzioni spagnole della Letteratura italiana PB98-1237.

2. Marcelino MenÉndez Pelayo, Estudio sobre Castiglione y El Cortesano, premesso alla ristampa della traduzione di Boscán a cura di Ángel GonZÁlEZ PALENCIA (Anejo XXV della "Revista de Filología Española»), Madrid, 1942, p. LIII.

3. Los quatro libros del cortesano, compuestos en italiano por el conde Balthasar castellón y agora nuevamente traduzidos en lengua castellana por Boscán... con privilegio imperial por diez 
glione naturalizzandolo attraverso la scorrevolezza della lingua. Ma dietro l'illusione di quella facile naturalezza si occultavano complesse tecniche di pedagogia interlineare miranti a ridurre l'impatto delle novità. Esse consistettero fondamentalmente nell'alternanza di due metodi traslativi opposti: quello volto a ricondurre vocaboli estranei a idee comuni (spesso arricchite di commenti o di giunte lessicali), e quello mirante a inoculare la nuova medicina ideologica nel tessuto verbale consueto orientandone in vario modo la risemantizzazione. Di qui l'oculato equilibrio fra traduzione e ricreazione riscontrato dagli studiosi di Boscán, e la tendenza a evitare «la rigidez de unas equivalencias rutinarias». ${ }^{4} \mathrm{Ne}$ fu consapevole il traduttore stesso, che espose inequivocabilmente il proprio criterio alla destinataria dell'opera:

Yo no terné fin en la traducción de este libro a ser tan estrecho que me apriete en sacalle palabra por palabra; antes, si alguna cosa en él se ofreciere que en su lengua parezca bien y en la nuestra mal, no dexaré de mudarla o de callarla. Y aun con todo esto he miedo que según los términos de estas lenguas italiana y española y las costumbres de entrambas naciones son diferentes, no haya de quedar todavía algo que parezca menos bien en nuestro romance (lettera-dedicatoria A la muy Magnífica Señora, Doña Jerónima Palova de Almogávar).

E confermò l'assunto Garcilaso, «presente a la postrera lima», in un'altra lettera-prefazione rivolta alla medesima nobildonna:

diose Boscán en esto tan buena maña que cada vez que me pongo a leer este su libro [...], no me parece que le hay escrito en otra lengua. Y si alguna vez se me acuerda del que he visto y leído, luego el pensamiento se me vuelve al que tengo entre las manos (A la muy Magnifica Señora, Doña Jerónima Palova de Almogávar).

años, Barcelona, por Pedro Mompetzat, 1534 (Colophon: «Aquí se acaban los cuatro libros del Cortesano, compuestos en italiano por el conde Baltasar Castellón y traducidos en lengua castellana por Boscán, imprimidos en la muy noble ciudad de Barcelona por Pedro Mompezat, imprimidor, a dos del presente mes de abril, mil y quinientos treinta y cuatro»; Privilegio di Carlo V: «Dat. en nuestra villa de Monzón a XX dias de deciembre del año del nacimiento de Nuestro Señor, mil quinientos treinta y tres»). Ne seguirono almeno 15 edizioni tra il 1539 e il 1588 , la seconda delle quali (1540) introdusse la divisione del testo in capitoli: Libro llamado el cortesano traduzido agora nueuamente en nuestro vulgar Castellano por Boscan. Con acotaciones por las margines, Salamanca, por Pedro Tovans, a costa de Guillermo de Millis [Colophon: "Aquí se acaba el libro llamado El cortesano del conde Baltasar Castellón, agora nuevamente corregido y enmendado con sus acotaciones por las márgenes. Impreso en Salamanca por Pedro Touans, a costa del honrado varón Guillermo de Milles. Acabose a quinze días del mes de henero, año de mil e quinientos y quarenta años»].

4. Sono parole di Margherita Morreale, nel suo ormai classico studio: Castiglione y Boscán: El ideal cortesano en el Renacimiento español, «Anejos del Boletín de la Real Academia Española», I 1959, p. 19. 
Ma questa insistenza nel miraggio linguistico era anche il segno di un desiderio più o meno inconsapevole di occultare la distanza salvata nella translatio delle idee. ${ }^{5}$ Garcilaso, insomma, voleva sottolineare, attraverso la riuscita dell'amico, la continuità semantica esistente fra il lessico di Castiglione e i «términos muy cortesanos y muy admitidos de los buenos oídos y no nuevos ni al parecer desusados de la gente» (ibidem). In realtà egli sapeva di aver importato un modello antropologico difforme dalla tradizione cavalleresca spagnola, che rischiava di mimetizzarvisi subdolamente pervertendone il significato. Lo dimostrò l'equivoco in cui era incorso Carlo V quando, volendo celebrare Castiglione alla sua morte, lo fece passare per un perfetto "cavaliere» con la conseguente idea annessa del perfetto vassallaggio: "Yo os digo que es muerto uno de los mejores caballeros del mundo». I tempi necessari all'assimilazione del nuovo erano, infatti, più lunghi del previsto; tant'è vero che il superamento ideologico del retroterra medievale avvenne solo con la crisi dell'Impero e si espresse nell'ironico linguaggio di Cervantes.

Valga questa premessa a introdurre qualche dubbio sull'assimilazione delle idee rinascimentali da parte di Boscán, il quale incorse in un lapsus meno clamoroso di quello di Carlo V, ma non meno significativo. Si trattò, infatti, di uno strano scambio di genere grammaticale nella parte dedicata a descrivere la perfetta donna di palazzo, e la svista cadde concretamente sulla disputa intavolata da Lorenzo il Magnifico e Gasparo Pallavicino in merito al contegno da tenere di fronte alle profferte galanti (III $\$ 56$ ), un caso in cui il Magnifico consigliava la donna di «rifiutar di creder sempre che chi le parla d'amore, l'ami però», mentre Gasparo la spingeva a riamare «quando conosce veramente esser amata» :

Non volete voi, signor Magnifico, che questa vostra così eccellente donna essa ancora ami, almen quando conosce veramente esser amata? Atteso che se 'l cortegiano non fosse redamato, non è già credibile che continuasse in amare lei e così le mancheriano molte grazie, e massimamente quella servitù e reverenzia, con la quale asservono e quasi adorano gli amanti la virtù delle donne amate.
Decidme, señor Manífico, ¿no os parecería a vos bien que esta vuestra tan ecelente dama amase a lo menos cuando verdaderamente se conociese ser amada? Considerando que si a nuestro cortesano le fuese mal con ella, está en la mano desgustarse luego, y dexar de servilla, y desta manera perdería $e ́ l$ muchas cosas buenas, las cuales ternía todas con gran abundancia, amándola; y entre las otras faltalle ía una muy sustancial, y sería aquella sojución y acatamiento con que acatan y casi adoran los enamorados a sus damas.

5. Di questa difficoltà dà conto l'alto numero di vocaboli problematici annoverati da M. Morreale, la quale si vide costretta a creare una sezione ad hoc nell'appendice per contenere la varia e numerosa casistica di deviazioni registrata: «son tantas las palabras que le causan resistencia o vacilación y las que le sugieren alguna glosa o comentario que, aun sin la menor 
Come vediamo, nel tradurre la replica di Pallavicino, Boscán confuse un dativo femminile (le) con un pronome maschile, talché là dove il malizioso misogino sconsigliava la donna di rifiutare le profferte dell'amante per non perdere, insieme al suo amore, le piacevoli manifestazioni dell'omaggio, Boscán assunse il punto di vista dell'innamorato immaginandolo privo «di molte cose buone che avrebbe potuto avere amandola» (perdería él muchas cosas buenas, las cuales ternía todas con gran abundancia, amándola).

Né pare casuale l'equivoco se si guarda alla controreplica del Magnifico avversa agli amori extramatrimoniali, perché essa appare amplificata da Boscán in un vero e proprio excursus inneggiante la forza incontenibile della passione, che adotta ancora una volta, per quanto in modo più coperto, la prospettiva maschile. Sicché mentre Castiglione fa pronunciare a Lorenzo parole recise di rifiuto ("lo amar come voi ora intendete estimo che convenga solamente alle donne non maritate»), la traduzione introduce un inciso ipotetico che ne offusca il nitore ("no lo ha de hacer [...], si se ha de tratar esa materia de amores con argumentos, sino que la que cayera caya, y la otra que se esté = 'non deve farlo... se di questa materia occorre discorrere con argomentazioni, ma quella che deve cadere, cada, e l'altra si tenga'):

Di questo - rispose il Magnifico - non la voglio consigliare io; dico ben che lo amar come voi ora intendete estimo che convenga solamente alle donne non maritate.

Eso que habéis preguntado, respondió el Magnífico, no lo ha de hacer ella por consejo, si se ha de tratar esa materia de amores con argumentos, sino que la que cayera caya, y la otra que se esté. Cosa que trae consigo una pasión tan grande como es amar, no se puede ordenar ni medir en los hombres ni en las mujeres, acaecimientos son o dolencias que es cosa difícil prevenillas, $y$ casi imposible curallas. Séos bien decir, si esto se ha de hablar por rigor de derecho y hemos de andar aquí en dotrinas y filosofias estrechas, que ese amar, como vos entendéis que sea, quizá no será lícito sino a las que están por casar.

Come a dire: 'non è cosa da risolvere in teoria, ma da lasciare a ciascuna donna secondo le forze sue, visto che la passione è incontenibile nei due sessi' (no se puede ordenar ni medir en los hombres ni en las mujeres); col che il divieto si attenua in un possibilista "forse» ("quizá no será lícito sino a las que están por casar»), limitando l'equiparazione uomo-donna al piano della passione medievalmente concepita come creaturale propensione a «cadere».

Questa tendenza di Boscán a tingere di antichi pregiudizi l’ideale di razionale contenutezza tratteggiato da Castiglione, viene confermata nel dibatti-

intención de hacer una concordancia, he tenido que ponerlas aparte y relegarlas a los apéndices», op. cit., p. 71. 
to successivo sul diritto delle donne sposate ma infelici a farsi amare da altri (III $\$ 56-57$ ); un caso in cui la divergenza di pareri fra Gasparo e Lorenzo si fa più sfumata, giacché, mentre il primo intona a una maggiore razionalità la resa della donna, il secondo ammette la legittimità di un amore platonico pur negando il rapporto carnale («voglio che niuna altra cosa allo amante conceda eccetto che l'animo»). Boscán doveva, insomma, misurarsi con due tesi parimenti liberali anche se in diverso grado. Da un lato, l'argomento di Pallavicino in difesa di un amore completo («non amando chi ama loro, fanno ingiuria a se stesse»), non presentava più il cedimento come piacevole "peccato», bensì come un caso di giustizia proporzionale, essendo l'amore altrui specchio dell'amor proprio; dall'altro, la controproposta del Magnifico (III \$ 57) mirava a dimostrare il carattere amabile dell'onestà muliebre conciliando i valori opposti della "grazia» e della "venustà» ("con la venustà, con la grazia induca nell'animo di chi la vede quell'amor vero che si deve a tutte le cose amabili»). Boscán doveva, insomma, aggirare due diverse trasgressioni del codice: l'estetica della morale e la legittimazione giuridica del piacere. Nel primo caso egli ci riuscì capovolgendo la proposta di amare chi ama nel comando eufemistico (litote) di non amare chi «non ama» ("pero las otras tampoco deben ofenderse a sí mismas, amando a quien no las ama»); nel secondo, intorbidando l'assunto neoplatonico con un excursus sui sublimi «tormenti» della rinuncia maschile per far regredire l'equilibrio fra morale e piacere verso il topos dell'assenza:

Però voglio che la mia donna di palazzo non con modi disonesti paia quasi che s'offerisca a chi la vole ed uccelli [...] ma con i meriti e virtuosi costumi suoi, con la venustà, con la grazia induce nell'animo di chi la vede quello amor vero che si deve a tutte le cose amabili e quel rispetto che leva sempre la speranza di chi pensa a cosa disonesta. Colui adunque che sarà da tal donna amato, ragionevolmente devrà contentarsi d'ogni minima dimostrazione ed apprezzar più da lei un sol sguardo con affetto d'amore che l'esser in tutto signor d'ogni altra
Gane ella hombres de bien por servidores [...] non con las artes que hemos dicho de las otras, sino con su gentileza, con sus buenas costumbres, con su autoridad, con su gracia, con un buen descuido, $y$, en fin, con decir y hacer lo que debe. Con estas cosas será ella amada y tenida en mucho, y mucho más en ausencia, $y$ desto nacerá, que el que se viese ser amado de una dama de tan gran precio, fácilmente sufrirá sus trabajos; y aunque muchas veces, de muy apretado de sus fatigas, venga a romper y casi a desperarse, todavia volverá sobre sí, $y$ hallará que tiene razón de contentarse, o a lo menos de sufrirse con cualquier señal de amor que en ella vea, por pequeña que le parezca, y preciará más una blandura o un buen mirar desta, que ser totalmente señor de otra

L'amore cortese si sovrappose così a quello cortigiano facendo emergere in contraluce una tesi oscillante fra condizione creaturale e sublimazione stilnovistica, la prima nella prospettiva dei due sessi, la seconda in quella maschile. 
Nell'un caso e nell'altro spariva la reciprocità del rapporto amoroso e la mediazione dialogica fra gli opposti.

L'esemplificazione si potrebbe allargare ad altri casi e ad altri temi, ma questi campioni varranno forse a mostrare come Boscán non fosse riuscito ad assimilare appieno l'erotica dell'ethos che andava tramandando. Un ideale riassunto da Castiglione nella figura femminile della modestia temperata (III \$ 57), e sulla cui 'difficoltà' si era soffermato all'inizio del Terzo Libro: «e però le bisogna tener una certa mediocrità difficile e quasi composta di cose contrarie, e giunger a certi termini a punto, ma non passargli» (III $\$ 5$ ). La difficoltà non risiedeva, quindi, soltanto nell'esecuzione del modello da parte delle donne, ma anche nella sua comprensione da parte degli uomini chiamati a completarne l'opera. Tale il valore del dialogo rinascimentale quale indispensabile forma di arbitraggio tra fra gli opposti. Lo scambio amoroso ne era la quintessenza.

\section{Bibliografia selettiva}

\section{Edizioni cinquecentesche della traduzione di Boscán}

Los quatro libros del cortesano, compuestos en italiano por el conde Balthasar castellon y agora nueuamente traduzidos en lengua castellana por Boscan... con privilegio Inperial por diez años, Barcelona, por Pedro Mompetzat, 1534, $114 \mathrm{ff}$.

Los quatro libros del Cortesano, compuestos en ytaliano por el conde Baltasar Castellón agora nueuamente traduzidos en lengua Castellana por Boscán, Toledo, s.i., 1539, 199 ff.; $4^{\mathrm{o}}$.

Libro llamado el cortesano traduzido agora nueuamente en nuestro vulgar Castellano por Boscan. Con sus acotaciones por las margines, Salamanca, por Pedro Tovans, a costa de Guillermo de Millis, 1540, 144 ff.; 40.

Libro llamado el cortesano traduzido agora nuevamente en nuestro vulgar castellano por Boscan, s.l. s.i. [Sevilla, Jacobo Cromemberg], 1542, 140 ff.; $4^{\circ}$.

Libro llamado el Cortesano tradvzido agora nueuamente en nuestro vulgar castellano por Boscan, Anueres, en casa de Martin Nucio, 1544, 239 ff.; $8^{\circ}$.

Libro llamado el cortesano traduzido agora nueuamente en nuestro vulgar Castellano por Boscan, Sevilla, Jacobo Cromemberg, 1549, 140 ff.; $4^{\circ}$.

Libro llamado el cortesano traducido en nuestro vulgar Castellano por Boscan, Zaragoza, a costa de Miguel Çapilla, 1553, $216 \mathrm{ff}$.; $8^{\circ}$.

Los quatro libros del Cortesano, compuestos en italiano por el conde Baltasar Castellón agora nueuamente traduzidos en lengua Castellana por Boscán, Toledo, s.i., 1559, 199 ff.; $4^{\mathrm{o}}$.

El Cortesano traduzido por Boscan en nuestro vulgar castellano nueuamente agora corregido, Anvers, en casa de la biuda de Martin Nutio, 1561, 247 ff.; $8^{\circ}$.

El Cortesano. Tradvzido de Italiano en nuestro vulgar castellano, por Boscan, Valladolid, Francisco Fernández de Córdoba, 1569, [8], 294 ff.; 8º.

El Cortesano traducido por Boscán en nuestro vulgar castellano nuevamente agora corregido, en Anvers, en casa de la biuda de Martin Nucio, 1571, 247, [3] ff.; $8^{\circ}$.

El Cortesano traducido por Boscán en nuestro vulgar castellano nuevamente agora corregido, Anvers, en casa de Philippo Nucio, 1574, 247, [3] ff.; 8.

El Cortesano traducido por Boscán en nuestro vulgar castellano nuevamente agora corregido, Anvers, en casa de Philippo Nucio, 1577, 247, [3] ff.; 8º. 
El cortesano. Traduzido de Italiano en nuestro vulgar Castellano por Boscan, Salamanca, en casa de Pedro Lasso, 1581, 290 [i.e. 304] ff.; 80.

El Cortesano traducido por Boscán en nuestro vulgar castellano nuevamente agora corregido, Anvers, en casa de Philippo Nucio, 1588, 247, [3] ff.; $8^{0^{6}}$.

\section{Studi sulla traduzione e sulla sua fortuna}

Burke, Peter. The Fortunes of the "Courtier». Cambridge: Polity Press, 1995, trad. cast. Los avatares del "Cortesano». Barcelona: Gedisa, 1998.

Corominas, Juan María. Castiglione y la Araucana: estudio de una influencia. Madrid: José Porrúa Turanzas, 1980.

FABIÉ, Antonio María. Introduzione a El Cortesano. Madrid: Librería de los Bibliófilos, 1873, p. I-LXIX.

Green, Otis H. Boscán and «Il cortegiano»: the "Historia de Leandro y Hero». Bogotá: Instituto Caro y Cuervo, 1948.

Hamilton, Rita. «Boscán traductor del «Cortesano» de Castiglione», in Boletín de la Academia Argentina de Letras, XXII (1957), p. 109-132, 231-329, 587-667.

KREBS, Ernest. "El "Cortesano» de Castiglione en España», in Boletín de la Academia Argentina de Letras, VIII (1940) 93-146 e 423-435; IX (1941) 135-142 e 517-543; X (1942) 53-118 e 689-748; XXII (1957), p. 109-132, 231-329, 587-667.

MENÉNDEZ Y PElaYO, Marcelino. "Juan Boscán» in Id., Antología de poetas líricos castellanos, X. Madrid: CSIC, 1945.

ID. Estudio sobre Castiglione y el Cortesano, in El Cortesano, a cura di Angel González Palencia. Madrid: Instituto Antonio de Nebrija, 1942, p. VII-LXIV.

Molinaro, J. Antonio. "Boscán's Translation of "Il Cortegiano" and his Linguistic Devices», in Quaderni Ibero-Americani, III (1959), p. 584-591.

Morreale, Margherita. Castiglione y Boscán: El ideal cortesano en el Renacimiento español (estudio léxico-semántico). Madrid, 1959 (Anejo del Boletín de la Real Academia Española, I). E cfr. rec. di Ghino Ghinassi in Lingua nostra, XXII (1961), p. 58-60.

NAVARRETE, Ignacio. "The Spanish Appropiation of Castiglione», in Yearbook of Comparative and General Literature, 39 (1990-1991), p. 35-46.

PozzI, Mario (a cura di); B. Castiglione. El Cortesano (edizione di Los quatro libros: del cortesano compuestos en italiano por el conde / Balthasar / castellon / y / agora nueuamente traduzidos / en/ lengua castellana por Boscan). Madrid: Cátedra, 1994 («Letras Universales»).

SAROLLI, Gian Roberto. "Boscán as translator: St. Jerome or the Humanists?», in Modern Language Notes, LXXVII (1962), p. 187-191.

TERRACINI, Lore. "Valdés: cuidado. Boscán: descuido», in Studi di letteratura spagnola, (1965), p. 187-209, poi in Id., Lingua come problema nella letteratura spagnola del Cinquecento. Torino: Stampatori, 1979.

TORRE, Esteban. "Garcilaso y Boscán en la historia de la traductología española", in J. César Santoyo (a cura di), Fidus interpres, I, Universidad de León, 1987, p. 145-155.

6. Non è stato possibile verificare l'esistenza di altre stampe registrate in diversi cataloghi (cfr. ad esempio: Bartolomé José GAYANGOS, Ensayo de una biblioteca de libros raros y curiosos, Madrid, 1866, vol. II, coll. 1447-1455; Antonio María FABIÉ e Ángel GONZÁlEz PALENCIA nelle edd. del Cortesano curate rispettivamente nel 1773 e nel 1942): Valencia, Juan de Arcos, 1561; Anvers, Nucio, 1599. 
3. Studi sulla figura della donna nel 'Cortegiano' di Castiglione

FINUCCI, Valeria. The lady Vanishes: subjectivity and representation in Castiglione and Ariosto, Standford: Standford University Press, 1992.

SACCARO BATTISTI, Giuseppa. «La donna, le donne nel Cortegiano», in Carlo Ossola (a cura di), La Corte e il Cortegiano, I: La scena del testo, 1980, p. 219-250.

ZanCAN, Marina. «La donna nel Cortegiano di B. Castiglione. Le funzioni del femminile nell'immagine di corte», in Id., (a cura di), Nel cerchio della luna. Figure di donna in alcuni testi del XVI secolo. Venezia: Marsilio, 1983, p. 13-56. 